\title{
Long non-coding RNA TUG1 recruits miR-29c-3p from its target gene RGS1 to promote proliferation and metastasis of melanoma cells
}

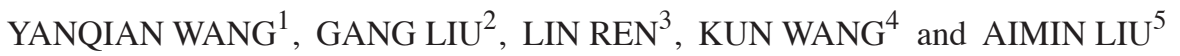 \\ ${ }^{1}$ Department of Dermatology, Xinxiang Central Hospital, Xinxiang, Henan 45300; ${ }^{2}$ Department of Dermatology, \\ Jiaozuo People's Hospital, Jiaozuo, Henan 454001; ${ }^{3}$ Wound Diagnosis and Treatment Center, Xinxiang Central Hospital, \\ Xinxiang, Henan 45300; ${ }^{4}$ Department of Dermatology, Jiaozuo Second People's Hospital, Jiaozuo, Henan 454000; \\ ${ }^{5}$ Department of Dermatology, Henan Traditional Chinese Medicine Hospital, Zhengzhou, Henan 450002, P.R. China
}

Received April 27, 2018; Accepted September 10, 2018

DOI: $10.3892 /$ ijo.2019.4699

\begin{abstract}
Melanoma is an aggressive type of skin cancer, characterized by high mortality rates worldwide. Therefore, the identification of new diagnostic markers and therapeutic targets for melanoma is imperative. Accumulating evidence has demonstrated that long non-coding RNAs (lncRNAs) play important roles in tumor initiation and progression. It was recently reported that the expression of lncRNA taurine upregulated 1 (TUG1) was relatively higher in cancer compared with that in normal cells, and that TUG1 promoted the progression of various cancers. However, the pattern of expression and mechanism of action of TUG1 in melanoma remain unclear. The aim of the present study was to investigate whether TUG1 expression is relatively higher in melanoma tissues and whether this expression is correlated with poor overall survival. Knockdown of TUG1 was found to suppress melanoma cell growth and metastasis and induce cell apoptosis. By contrast, the overexpression of TUG1 promoted the growth and metastasis of melanoma cells, and inhibited their apoptosis. In addition, the results of the present study indicated that TUG1 sequestered endogenous miR-29c-3p and that it was able to suppress its expression. Furthermore, it was observed that miR-29c-3p could reverse the promoting effect of TUG1 on melanoma progression, which may be associated with the positive regulation of regulator of G-protein signaling 1 (RGS1), a target
\end{abstract}

Correspondence to: Dr Aimin Liu, Department of Dermatology, Henan Traditional Chinese Medicine Hospital, 6 Dongfeng Road, Zhengzhou, Henan 450002, P.R. China

E-mail: aimin_liu128@hotmail.com

Abbreviations: TUG1, taurine upregulated gene 1; RGS1, regulator of G-protein signaling 1; WT, wild-type; MT, mutant

Key words: long non-coding RNA, taurine upregulated 1, miR-29c-3p, regulator of G-protein signaling 1 gene of miR-29c-3p. Taken together, the data of the present study demonstrated that TUG1 promoted proliferation and invasion and suppressed apoptosis in melanoma cells by regulating miR-29c-3p and its target gene, RGS1. Therefore, lncRNA TUG1 appears to be a promising diagnostic marker for melanoma patients.

\section{Introduction}

Melanoma is a skin malignancy with a high mortality rate (1) and an increasing incidence worldwide (2-4). Therefore, the development of novel antineoplastic agents and the investigation of their underlying mechanisms are imperative in the treatment of this malignant tumor.

Long non-coding RNAs (lncRNAs) generally consist of $>200$ nucleotides and do not encode proteins (5). XIST was the first documented functional lncRNA identified in the early 1990s (6). Initially, lncRNAs were generally considered as transcriptional noise or useless sequences (7). However, recent studies have demonstrated that lncRNAs play important roles in several biological processes, including dosage compensation, regulation of gene expression, genomic imprinting, nuclear organization and compartmentalization. Changes in the expression of IncRNAs are associated with the occurrence of several diseases, including psoriasis, Alzheimer's disease, heart disease and cancer. A growing body of evidence indicates that lncRNAs play an important role in the diagnosis and prognosis of various malignancies, including clear cell renal cell carcinoma (8), glioma (9) and osteosarcoma (10). Recently, accumulating evidence suggests that IncRNAs are also implicated in the progression and prognosis of melanoma (11-14). Of note, the expression and distribution of lncRNAs differ according to the cell type to match their possible regulatory role.

Taurine upregulated 1 (TUG1) is a novel lncRNA located on chromosome $22 \mathrm{q} 12$, which is composed of 6.7-kb nucleotides (15). Previous reports have demonstrated that TUG1 lncRNA is largely overexpressed and positively regulates oncogenesis in various types of cancer, such as glioma (16), esophageal squamous cell carcinoma (17), 
endometrial and colorectal cancer $(18,19)$, hepatocellular carcinoma (20) and osteosarcoma (21). However, in certain cancers, such as non-small-cell lung cancer, TUG1 has been reported to be expressed at a relatively low level and acts as a tumor suppressor (22). These reports indicate that TUG1 may play different roles in different types of cancer cells, and TUG1 activity may change under different tumor microenvironment conditions.

The data of the present study demonstrated that TUG1 was highly expressed in human melanoma tissues and cell lines. Moreover, TUG1 facilitated cell proliferation and invasion and suppressed cell apoptosis by sequestering miR-29c-3p from its target gene regulator of G-protein signaling 1 (RGS1) in melanoma cells. The results indicate that TUG1 may be promising as a potential diagnostic marker and therapeutic target for melanoma.

\section{Materials and methods}

Ethics statement. The present study was approved by the Ethics Committee of the Xinxiang Central Hospital (Xinxiang, China). The sample collection and surgical procedures were not harmful to the patients. Written informed consent was obtained from all patients. The research protocol conformed to the principles outlined in the Declaration of Helsinki.

Clinical samples. Between March 2014 and November 2017, a total of 40 melanoma tissues, 30 benign nevi and 15 metastatic melanoma tissues were obtained from patients who presented at the Xinxiang Central Hospital (Xinxiang, China). The patients had never received chemotherapy or radiotherapy prior to surgery. All the samples were immediately snapfrozen in liquid nitrogen and stored at $-80^{\circ} \mathrm{C}$ prior to RNA extraction.

RNA extraction and reverse transcription-quantitative polymerase chain reaction $(R T-q P C R)$ analysis. TRIzol reagent (Thermo Fisher Scientific, Inc., Waltham, MA, USA) was used to extract total RNA from the cells or the tissue samples. The RNA purity was confirmed by the A260/A280 ratio. cDNA was synthesized by reverse transcription using the TaqMan ${ }^{\circledast}$ MicroRNA Reverse Transcription kit (Thermo Fisher Scientific, Inc.) in accordance with the manufacturer's instructions. TaqMan ${ }^{\circledR}$ Universal PCR Master Mix Kit (Thermo Fisher Scientific, Inc.) was used to amplify the products by PCR. The relative levels were calculated using the $2^{-\Delta \Delta \mathrm{Cq}}$ method. The primers were purchased from RiboBio Co. Ltd. (Guangzhou, China). The primer sequences were as follows: TUG1 forward: 5'-AACTACCTGGACCGCTTCCT-3' and reverse: 5'-CCAC TTGAGCTTGTTCACCA-3'. miR-29c levels were detected by TaqMan microRNA assay (Applied Biosystems; Thermo Fisher Scientific, Foster City, CA, USA).

Cell culture and cell transfection. The human melanoma cell lines A375 and SK-MEL-2 were obtained from the American Type Culture Collection (Manassas, VA, USA). The cells were routinely cultured in complete Dulbecco's modified Eagle's medium (Gibco; Thermo Fisher Scientific, Grand Island, NY, USA) with 10\% fetal bovine serum (FBS; Gibco; Thermo Fisher Scientific), $100 \mathrm{U} / \mathrm{ml}$ penicillin and $100 \mu \mathrm{g} /$ ml streptomycin (Sigma-Aldrich; Merck KGaA, St. Louis, MO, USA). The cultured cells were routinely passaged every 2-3 days. All cell lines used were between passages 3 and 8 during the experiments.

According to the manufacturer's instructions, the cells were cultured in 6-well plates and transfected with $2 \mu \mathrm{g}$ of each plasmid in each well alone in the presence of $4 \mu 1$ Lipofectamine 200 (Invitrogen; Thermo Fisher Scientific, Carlsbad, CA, USA). TUG1, control RNA sequences and TUG1 siRNA (si-TUG1) were purchased from GenePharma (Shanghai, China). miR-29c-3p mimics, inhibitors, and the respective negative control (NC) were purchased from RiboBio (Guangzhou, China). The si-TUG1 sequence was GGTGGTTGAAAGGAATCCT. The TUG1 cDNA was amplified and subcloned into a pcDNA3.1 vector (Invitrogen; Thermo Fisher Scientific) in order to produce the TUG1 overexpression vector.

Cell Counting Kit-8 (CCK-8) assay. Cell proliferation was evaluated using CCK-8 (Dojindo, Kumamoto, Japan). After $48 \mathrm{~h}$ of transfection, 3,000 cells $(100 \mu \mathrm{l} /$ well) were seeded in 96-well plates. The cells were grown for 24,48 and $72 \mathrm{~h}$ and $10 \mu 1$ of CCK- 8 were added to each well. The samples were subsequently incubated at $37^{\circ} \mathrm{C}$ for $4 \mathrm{~h}$. A scanning multiwell spectrophotometer (Thermo Scientific, Inc.) was used to measure the absorbance at $450 \mathrm{~nm}$.

Flow cytometric analysis. After $48 \mathrm{~h}$ of transfection, the cells were detached using trypsin for $2 \mathrm{~min}$ at $37^{\circ} \mathrm{C}$, collected and rinsed twice with phosphate-buffered saline (PBS). The cells were counted and diluted to a density of 106 cells $/ \mathrm{ml}$. The apoptotic cells were verified by an Annexin V/FITC kit (KGA108, Nanking, China) according to the manufacturer's instructions. The collected cells were then centrifuged and the supernatants were discarded. The cells were resuspended in $200 \mu \mathrm{l}$ binding buffer. A total of $2 \mu \mathrm{l}$ of Annexin V-FITC solution and $5 \mu \mathrm{l}$ of $1 \mu \mathrm{g} / \mathrm{ml}$ propidium iodide were subsequently added to the cells. The cells were incubated in the dark at $37^{\circ} \mathrm{C}$ for $30 \mathrm{~min}$. The population of cells was then defined. FITC and $\mathrm{PI}^{-}$cells were designated as viable, $\mathrm{FITC}^{+}$and $\mathrm{PI}^{+}$cells were designated as late apoptotic or necrotic, and $\mathrm{FITC}^{+}$and $\mathrm{PI}^{-}$cells were designated as apoptotic. The results are representative of three independent experiments and each sample was run in triplicate.

Transwell invasion assays. The invasive ability of the cells was assessed by Transwell invasion assays (Corning Inc., Corning, NY, USA). Briefly, $8-\mu \mathrm{m}$ Transwell filter inserts were coated with Matrigel (10 mg/l, BD Biosciences, San Jose, CA, USA). Approximately $3 \times 10^{4}$ transfected cells were resuspended in $200 \mu \mathrm{l}$ serum-free medium and added onto $8-\mu \mathrm{m}$ Transwell filter inserts. A total of $500 \mu \mathrm{l}$ medium containing $20 \%$ fetal bovine serum were added to the lower chamber as a chemoattractant. Following incubation for $24 \mathrm{~h}$, the cells in the upper chamber were removed with a cotton swab. The cells invading to the lower surface of the membrane were fixed with methanol and then stained with $0.1 \%$ crystal violet solution for $10-20 \mathrm{~min}$ and the number of invading cells was counted. Each experiment was performed in triplicate. 
Western blot analysis. Cells $\left(\sim 1 \times 10^{7}\right)$ were collected and lysed in RIPA buffer (Beyotime Biotech, Nantong, China). This buffer was supplemented with protease (PMSF) and phosphatase inhibitors (Na-ortho-vanadate, NaF). Equal amounts of total protein were extracted from the cultured cells or tissues and separated by $8-10 \%$ SDS-PAGE. The samples were transferred onto polyvinylidene difluoride (PVDF) membranes. The PVDF membranes were incubated with primary antibodies overnight at $4^{\circ} \mathrm{C}$ : $\operatorname{RGS} 1(1: 1,000$, ab117077), Bcl-2 (1:1,000, ab196495) and MMP2 (1:1,000, ab37150). After washing, the PVDF membrane was incubated with horseradish peroxidase-conjugated secondary antibodies $(1: 5,000$, ab150077) at room temperature for $1 \mathrm{~h}$. Antibodies were purchased from Abcam (Cambridge, MA, USA). The secondary antibodies bound on the PVDF membrane reacted with ECL substrate (Pierce Chemical Co, Rockford, IL, USA) and the protein bands were exposed to $\mathrm{X}$-ray films. The results were normalized to the expression of the internal control $\beta$-actin.

Luciferase reporter assay. The human melanoma cell lines A375 and SK-MEL-2 were co-transfected with miR-29c-3p mimics or miR-control, pmiR-reporter luciferase vector containing a specific sequence of wild-type and/or a mutant TUG1 fragment. The transfection was achieved using Lipofectamine 2000 (Invitrogen; Thermo Fisher Scientific, Inc.). Following transfection and incubation for 48 h, the Dual-luciferase Reporter Assay System (Promega Corporation, Madison, WI, USA) was employed to evaluate the luciferase activities. The relative luciferase activity was normalized against the Renilla luciferase activity. Each experiment was performed in triplicate.

Pull-down assay. Pull-down assay was performed according to the manufacturer's instructions (Thermo Fisher Scientific, Inc.). Briefly, the biotinylated TUG1 probe or control probe were incubated with Dynabeads M-280 Streptavidin (Thermo Fisher Scientific, Inc.) after being dissolved in binding and washing buffer for $10 \mathrm{~min}$ at $25^{\circ} \mathrm{C}$, followed by generation of the probe-coated beads. Subsequently, the probe-coated beads were incubated with A375 or SK-MEL-2 cell lysates. Finally, the enriched RNA complexes in the beads were purified using TRIzol (Sigma-Aldrich; Merck KGaA) and detected using RT-qPCR. The TUG1 probe sequence was as follows: 5'-AAGA CTGAATCGGACTGCGTTAGA-3'; The negative control sequence was as follows: 5'-AAGACTGACCCAGACTTCA CAGCA-3'.

Immunohistochemical (IHC) staining. Standardized and automated IHC was used to detect the expression of RGS1 in benign nevi and melanoma tissues. Briefly, human benign nevi and melanoma tissues were fixed in $3 \%$ formaldehyde and embedded in paraffin. $0.3 \% \mathrm{H}_{2} \mathrm{O}_{2}$ was applied to block endogenous peroxidase activity. Subsequently, the sections were deparaffinized in a series of graded alcohols and microwaved in EDTA buffer for $10 \mathrm{~min}$ at $450 \mathrm{~W}$. Later on, the sections were washed with PBS and incubated with anti-RGS1 primary antibodies at $37^{\circ} \mathrm{C}$ for $30 \mathrm{~min}$. Horseradish peroxidase and diaminobenzidine were used as substrates to assess RGS1 expression.
Animal tumor model. BALB/c nude mice 7-8 weeks old were obtained from the Shanghai Laboratory Animal Center (Shanghai, China) and housed in barrier facilities with a 12-h light/dark cycle. TUG1-transfected A375 cells were subcutaneously injected into the right flank of the nude mice. The tumors were measured weekly and their volumes were calculated according to the equation: $\mathrm{V}=\left(\right.$ length $\mathrm{x}$ width $\left.{ }^{2}\right) / 2$. All animals were euthanized using $\mathrm{CO}_{2}$ after 4 weeks. The animal experiments were fully approved by the Ethics Committee of Animal Experiments of the Xinxiang Central Hospital. In addition, the present study was strictly performed in accordance with the recommendations in the Guide for the Care and Use of Laboratory Animals of the National Institutes of Health.

Statistical analysis. All the data are expressed as mean \pm standard deviation. The comparison between two groups was analyzed by Student's t-test. The comparisons among multiple groups were performed with one-way analysis of variance followed by Dunnett's test. A P-value $<0.05$ was considered to indicate statistically significant differences. The survival analysis was performed using Kaplan-Meier plots and log-rank tests. A P-value $<0.05$ was considered to indicate statistically significant differences.

\section{Results}

TUG1 expression in melanoma tissues and its association with overall survival. Recently, various studies indicated that overexpression of TUG1 may predict poor prognosis in cancer patients $(23,24)$. Therefore, the expression levels of TUG1 in melanoma tissues, benign nevi, primary melanoma tissues and metastatic melanoma tissues were detected by RT-qPCR assays. TUG1 was found to be overexpressed in melanomas compared with benign nevi (Fig. 1A). Moreover, the expression of TUG1 was upregulated in metastatic melanoma compared with primary melanoma tissues. Kaplan-Meier survival analysis and the log-rank test demonstrated that patients with high TUG1 expression exhibited significantly decreased overall survival (Fig. 1B, 27.3 vs. 57.9\%, respectively; hazard ratio $=2.44 ; 95 \%$ confidence interval: $1.57-3.78 ; \mathrm{P}<0.05)$. The data revealed that the expression levels of TUG1 in melanoma tissues was upregulated, which was in accordance with previous reports $(25,26)$. Furthermore, TUG1 expression was found to be positively associated with patient survival and cancer metastasis.

TUG1 expression in melanoma cells. To determine the expression of TUG1 in melanoma cells, TUG1 expression levels were initially investigated using RT-qPCR assays. TUG1 was found to be highly expressed in melanoma cell lines (Fig. 2A). Moreover, TUG1 was more highly expressed in SK-MEL-2 cells compared with A375 cells. A TUG1 overexpression plasmid and siRNA sequences against TUG1 were used and transfected to melanoma cell lines in order to investigate the role of this protein in melanoma.

Subsequently, the transfection efficiencies of TUG1 overexpression and knockdown were validated by RT-qPCR analysis. The transfection of TUG1 resulted in marked upregulation of TUG1 expression compared with the negative 
A

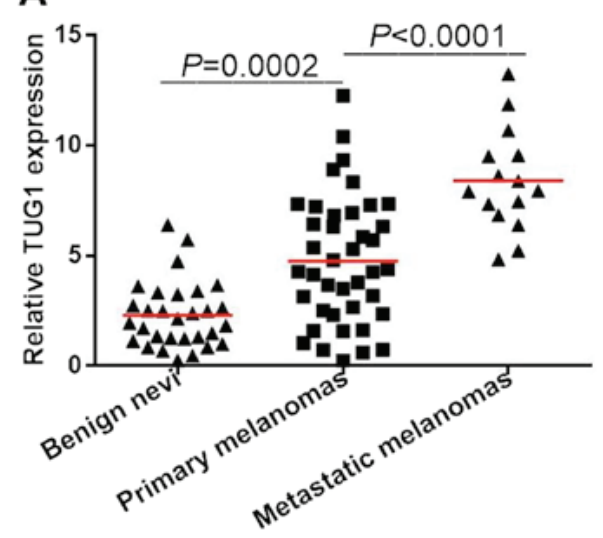

B

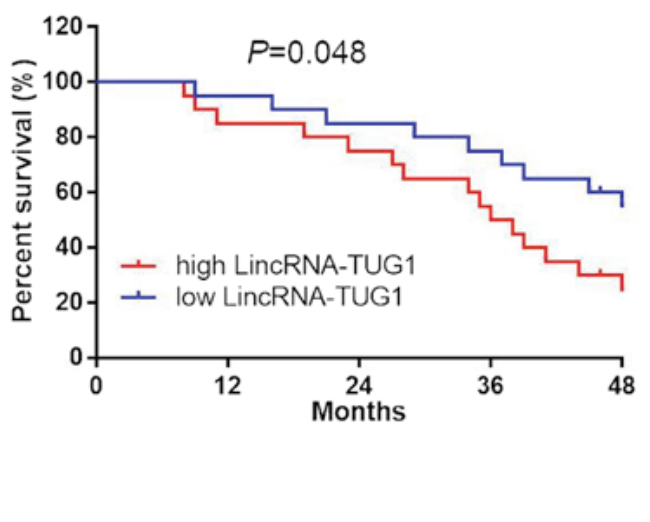

Figure 1. TUG1 expression in melanoma and the association with overall survival. (A) Relative expression of TUG1 in melanoma tissues, benign nevi, primary melanoma tissues and metastatic melanoma tissues. U6 was used as endogenous control. (B) Patients with high TUG1 expression exhibited poorer overall survival. TUG1, taurine upregulated 1.
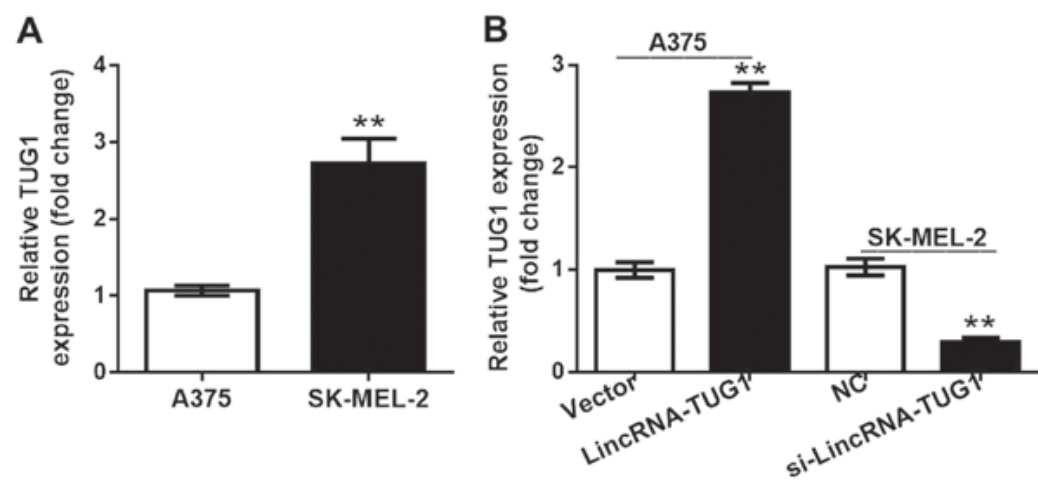

Figure 2. Relative expression of TUG1 in melanoma cell lines. (A) TUG1 expression was investigated in the human melanoma cell lines SK-MEL-2 and A375, with $\beta$-actin as an internal control. ${ }^{* *} \mathrm{P}<0.01$. The comparison was made by Student's t-test. (B) Transfection efficiency of pcDNA3.1-TUG1 in A375 cells and knockdown efficiency of TUG1 siRNA in SK-MEL-2 cells. The data are presented as the mean \pm standard deviation; ${ }^{* *} \mathrm{P}<0.01$. The comparisons were made by one-way analysis of variance followed by Dunnett's test. TUG1, taurine upregulated 1.

control (vector) in A375 cells (Fig. 2B). It was also observed that the expression of TUG1 was notably downregulated in SK-MEL-2 cells transfected with si-TUG1 in comparison with the transfection of non-specific siRNA (NC).

TUG1 regulates the proliferation, apoptosis and invasion of melanoma cells. It has been reported that the upregulation of TUG1 may promote the proliferation of cancer cells $(27,28)$, although its potential contribution to the pathogenicity of melanoma remains elusive. Therefore, the proliferation of melanoma cells was assessed using CCK-8 assays at 0, 24, 48 and $72 \mathrm{~h}$ following transfection. The overexpression of TUG1 significantly promoted A375 cell proliferation, while TUG1 knockdown significantly suppressed SK-MEL-2 cell proliferation (Fig. 3A). The effect of TUG1 on melanoma cell apoptosis was validated by flow cytometry. Overexpression of TUG1 suppressed the induction of apoptosis in A375 cells, whereas the depletion of TUG1 by si-TUG1 induced the apoptotic cascade in SK-MEL-2 cells (Fig. 3B). Similarly, the Transwell invasion assay indicated that TUG1 upregulation significantly induced the invasion of A375 cells, whereas knockdown of TUG1 significantly reduced the invasive capacity of SK-MEL-2 cells (Fig. 3C). As expected, the overexpression of TUG1 enhanced the proliferation and invasive ability of A375 cells, whereas the depletion of TUG1 suppressed the proliferation and invasive activity of SK-MEL-2 cells.

TUG1 negatively regulates the expression levels of $\mathrm{miR}-29 c-3 p$ and the levels of apoptosis-and invasion-related proteins. Various reports have demonstrated that TUG1 can recruit and modulate miRNAs that compete with the function and expression of competitive endogenous (ce)RNAs $(18,29)$. To investigate the interaction between TUG1 and miR-29c-3p, RT-qPCR assays were performed to determine the levels of the expression of miR-29c-3p. The data indicated that overexpression of TUG1 suppressed the expression of miR-29c-3p in A375 cells, whereas the depletion of TUG1 by si-TUG1 promoted the expression of miR-29c-3p in SK-MEL-2 cells (Fig. 4A, $\mathrm{P}<0.05$ ). Moreover, the application of miR-29c-3p mimics reversed the suppressive effect of TUG1 in A375 cells, and the miR-29c-3p inhibitor reversed the induction of SK-MEL-2 cells by si-TUG1 (Fig. 4B, P<0.05). The expression levels of the apoptosis-related protein $\mathrm{Bcl}-2$ and the invasion-related protein matrix metalloproteinase (MMP)2 were then examined. The overexpression of TUG1 

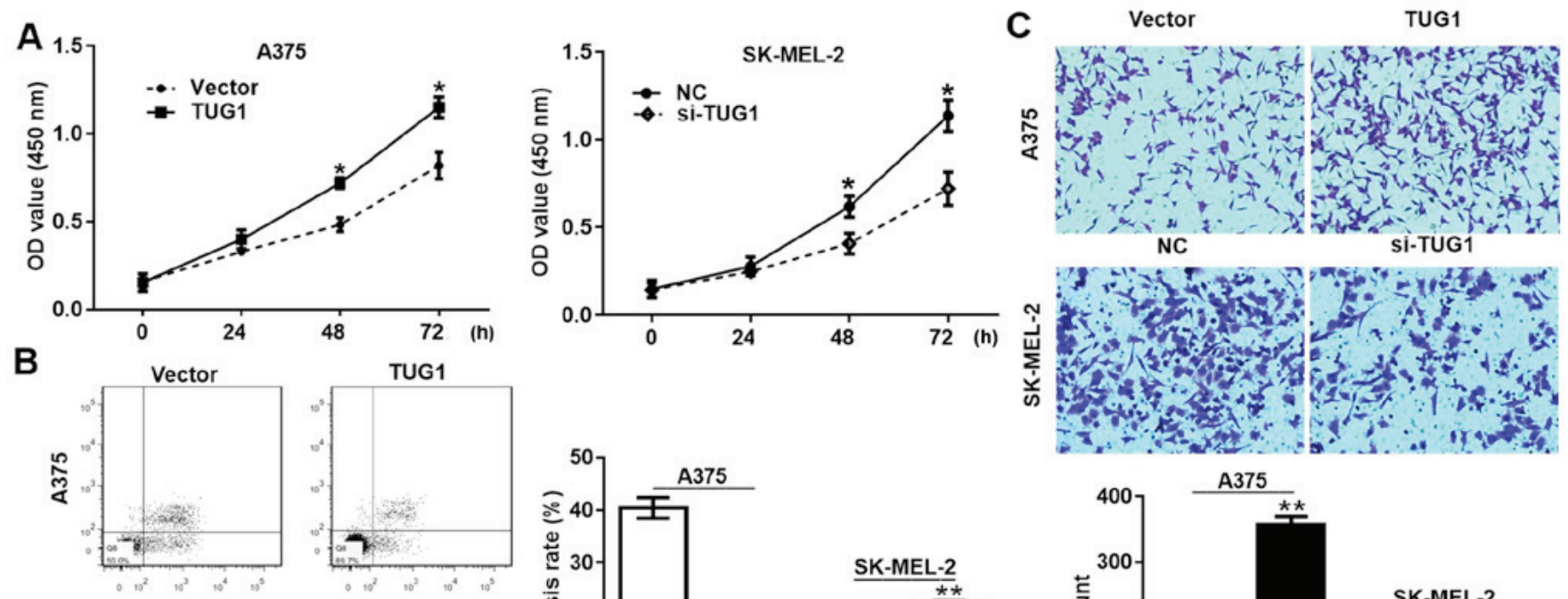

TUG1
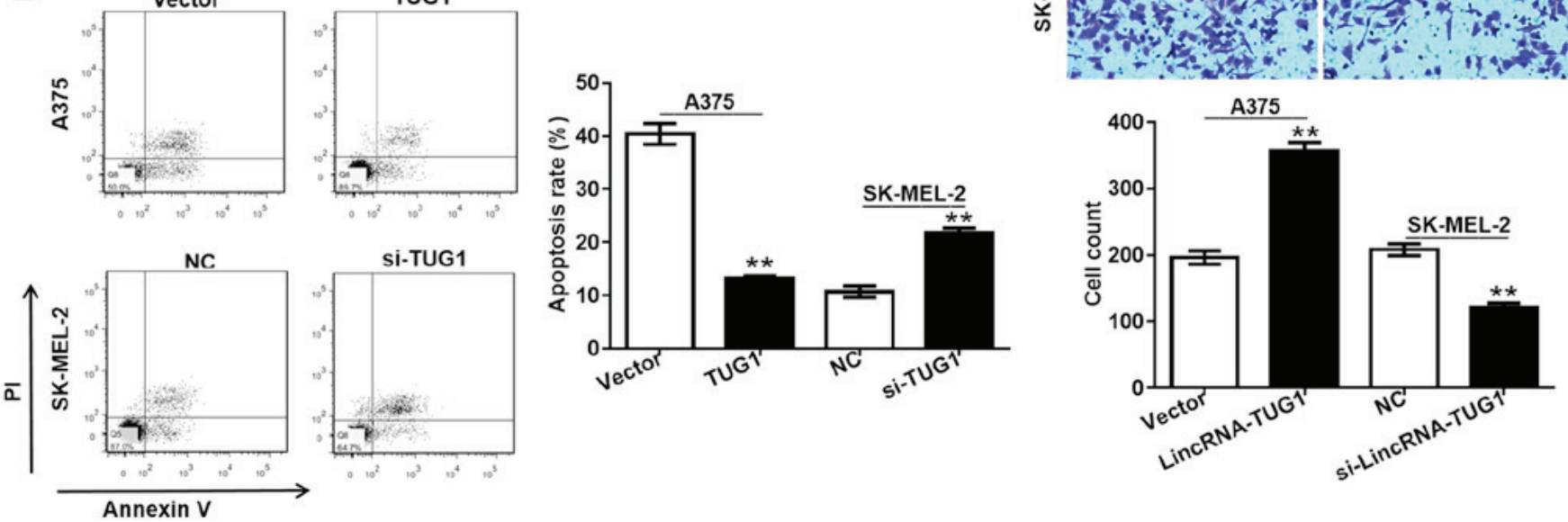

Figure 3. The regulation of TUG1 affects the proliferation and motility of melanoma cells. (A) The effect of TUG1 on cell proliferation was assessed by Cell Counting Kit-8 assays. ${ }^{* *} \mathrm{P}<0.01$. The comparison was made by Student's t-test. (B) The effect of TUG1 on apoptosis was assessed by Annexin V/FITC assay. Annexin V-positive cells were counted by flow cytometry. The graphs exhibit the apoptotic rate which was calculated as the number of Annexin V-positive cells/number of total cells x 100\%. A375 cells were seeded in 96-well plates and cultured in fetal bovine serum-free medium overnight prior to treatment. (C) Representative images of trastuzumab treatment on the invasion of mutant MCF7 cells in a Transwell invasion assay. The photographs were obtained using a magnification of $x 200$. The quantitative analysis of the number of invasive cells was determined by counting cells under a microscope. The data are presented as mean \pm standard deviation; ${ }^{* * *} \mathrm{P}<0.01$. The comparisons were made by one-way analysis of variance followed by Dunnett's test. TUG1, taurine upregulated 1 .

increased the expression of Bcl-2 and MMP2 in A375 cells (Fig. 4C and D). The depletion of TUG1 by si-TUG1 suppressed the expression levels of Bcl-2 and MMP2 in SK-MEL-2 cells. Moreover, miR-29c-3p mimics reversed the induction of A375 cells by TUG1. The miR-29c-3p inhibitor reversed the suppressive effect of si-TUG1 on SK-MEL-2 cells. These findings indicated that TUG1 negatively regulated the expression of miR-29c-3p and the expression levels of the apoptosis- and invasion-related proteins.

TUG1 promotes growth and invasion of melanoma cells via targeting $m i R-29 c-3 p$. To further confirm the regulatory effect of miR-29c-3p on TUG1, CCK8 assay was performed to evaluate cell viability. The CCK- 8 assay indicated that miR-29c-3p mimics could reverse the inductive effects of TUG1 on A375 cells, and miR-29c-3p inhibitor could reverse the inhibitory effects of si-TUG1 on melanoma cell proliferation (Fig. 5A). In addition, it was demonstrated that miR-29c-3p mimics increased the apoptotic rate of A375 cells compared with the TUG1 group (Fig. 5B). The application of the miR-29c-3p inhibitor reduced the apoptotic rate of SK-MEL-2 cells transfected with si-TUG1. Transwell assays indicated that treatment with miR-29c-3p mimics reduced considerably the number of A375 cells that invaded the lower chamber compared with NC cells (transfected with TUG1 plus miR-29c NC). The application of the miR-29c-3p inhibitor considerably increased the number of SK-MEL-2 cells that invaded the lower chamber compared with the NC cells (transfected with si-TUG1 plus miR-29c NC). Collectively, si-TUG1 exerted its anti-proliferative, anti-invasive and pro-apoptotic effects partly by targeting miR-29c-3p in melanoma cells.

RGS1 is a target of miR-29C-3p that is regulated by TUG1. In recent years, the majority of studies have demonstrated that lincRNA may modulate miRNAs as a ceRNA or molecular sponge (30). To determine whether TUG1 acts in a similar manner, the online software Starbase 2.0 was used to predict the miRNA target sites. Bioinformatic analysis indicated that miR-29c-3p had putative binding sites with TUG1 (Fig. 6A). Subsequently, dual luciferase reporter assays were performed in order to explore the interaction between miR-29c-3p and TUG1. The data indicated that TUG1 upregulated the luciferase activity of pmirGLO-TUG1. Moreover, miR-29c-3p mimics reversed the inductive effect of pmirGLO-TUG1, and reduced the luciferase activity of pmirGLO-TUG1 (Fig. 6B). In addition, western blot assays were used to identify whether TUG1 overexpression markedly improved RGS1 protein levels, while this effect of TUG1 on RGS1 expression was markedly reduced by miR-29c-3p mimics in A375 cells (Fig. 6C). In contrast to TUG1 overexpression, TUG1 knockdown caused a significant reduction in RGS1 protein expression and the miR-29c-3p inhibitor reversed the inhibitory effect of si-TUG1 on the expression levels of RGS1 in SK-MEL-2 cells (Fig. 6C). Furthermore, the pull-down assay indicated that 

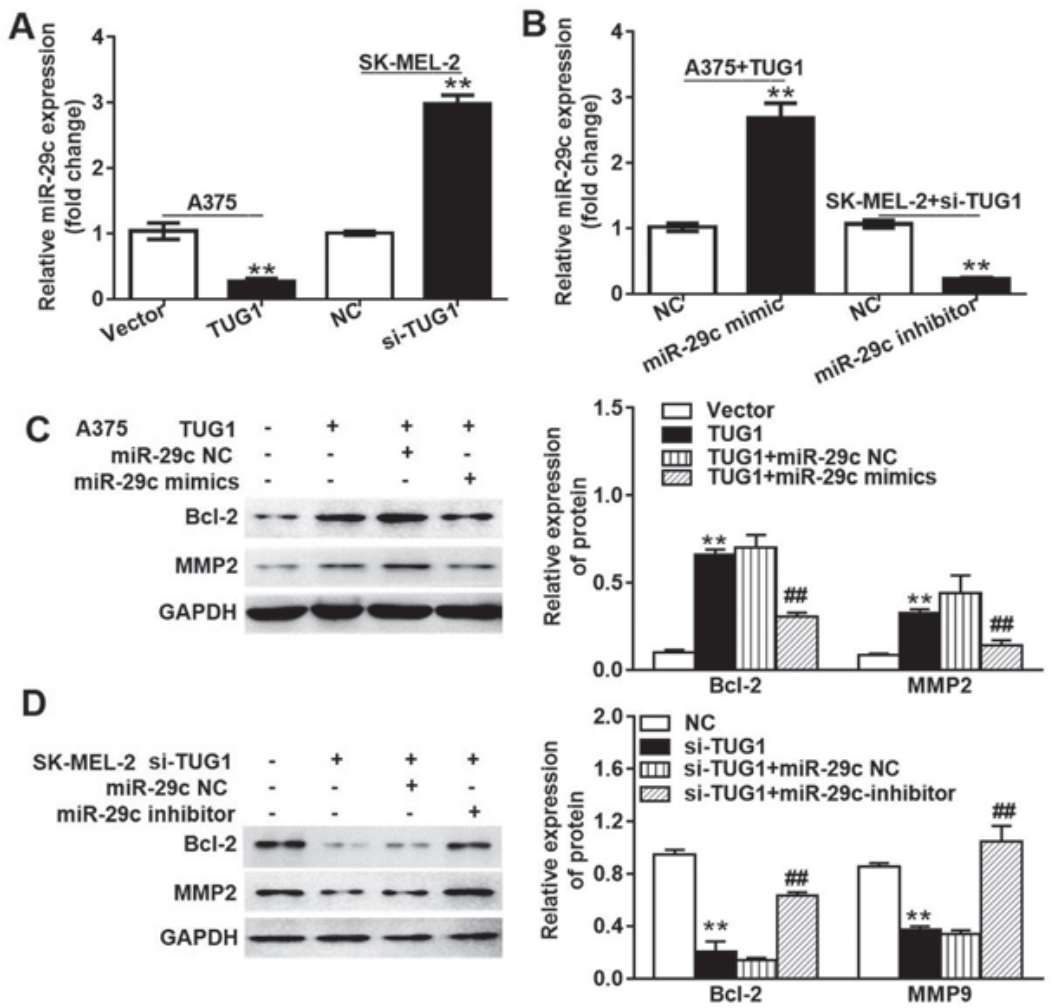

Figure 4. TUG1 downregulates the expression of miR-29c-3p and the expression levels of apoptosis-related and invasion-related proteins. (A) TUG1 downregulates the expression of miR-29c-3p. (B) miR-29c-3p mimics and miR-29c-3p inhibitor reversed the inductive effect of TUG1 and si-TUG1 on miR-29c-3p expression. (C) The cell lysates were immunoprecipitated and subjected to western blot analysis in order to detect the levels of the apoptosis-related and invasion-related proteins in A375 cells. (D) Western blotting was performed to detect the apoptosis-related and invasion-related proteins in SK-MEL-2 cells. Representative results are shown from three repeats with a similar pattern. The data are presented as the mean \pm standard deviation; ${ }^{*} \mathrm{P}<0.05,{ }^{* *} \mathrm{P}<0.01$ vs. vector group. ${ }^{\# \#} \mathrm{P}<0.01$ vs. TUG1 group. The comparisons were made by one-way analysis of variance followed by Dunnett's test. TUG1, taurine upregulated 1 .
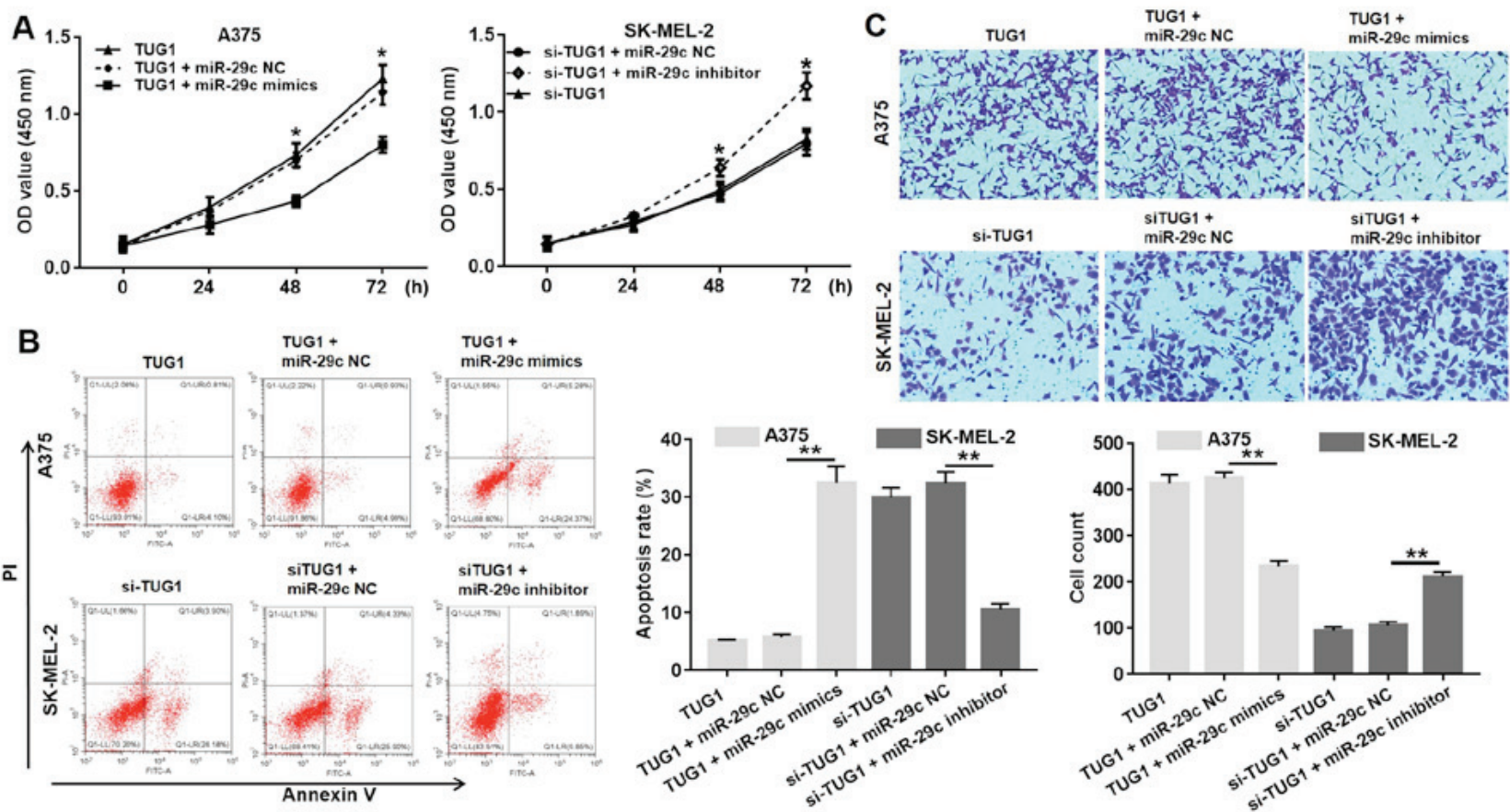

Figure 5. TUG1 promotes cell growth and invasion by targeting miR-29c-3p. (A) The effects of TUG1 and miR-29c-3p on cell proliferation were assessed by the Cell Counting Kit-8 assay. (B) Flow cytometry analysis indicated that miR-29c-3p mimics and miR-29c-3p inhibitor reversed the pro-apoptotic effect of TUG1 and si-TUG1 on melanoma cells. SK-MEL-2 cells were seeded in 96 -well plates and cultured in fetal bovine serum-free medium overnight prior to treatment. (C) Transwell invasion assay was used to explore the role of miR-29c-3p mimics and miR-29c-3p inhibitor in the regulation of invasive ability of melanoma cells transfected with TUG1 and si-TUG1. The photographs were captured under a magnification of x200. Representative results are shown from three repeats with a similar pattern. The data are presented as the mean \pm standard deviation; ${ }^{*} \mathrm{P}<0.05,{ }^{* *} \mathrm{P}<0.01$. The comparisons were made by one-way analysis of variance followed by Dunnett's test. TUG1, taurine upregulated 1. 
A

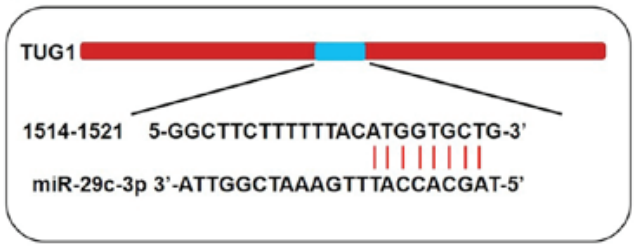

B

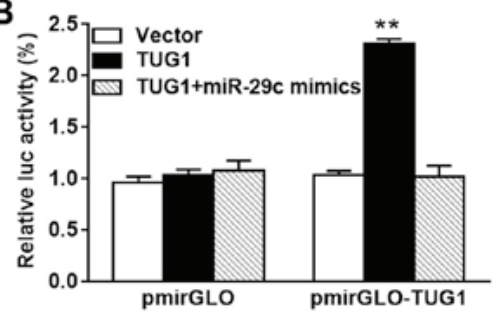

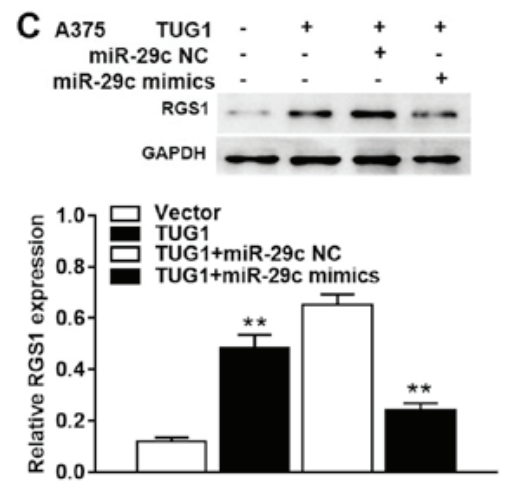
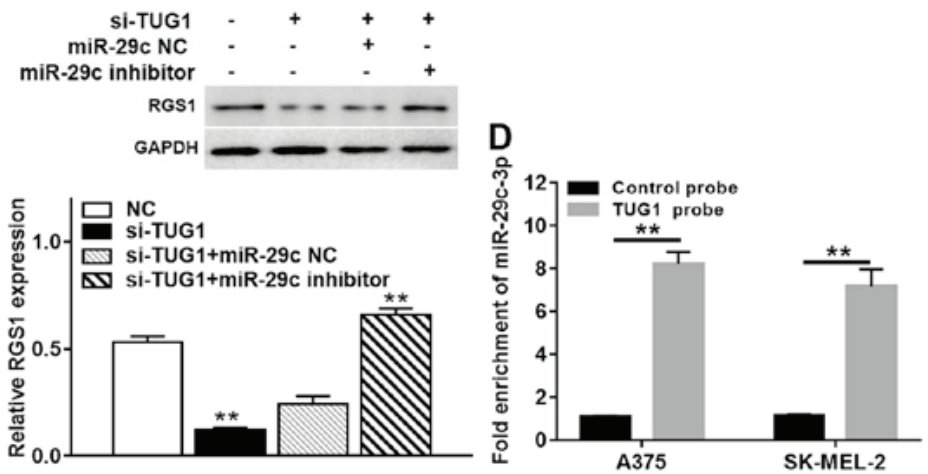

Figure 6. TUG1 recruits miR-29c-3p to facilitate RGS1 expression in melanoma cells. (A) The sequences of the predicted miR-29c-3p binding site and the TUG1 segments containing the wild-type binding site are shown. (B) Luciferase activity in cells co-transfected with vector or TUG1 or TUG1 + miR-29c-3p mimics, and luciferase reporters containing TUG or no specific promoter. (C) Melanoma cells were transfected with TUG1, TUG1 + miR-29c-NC, TUG1 + miR-29c-3p mimics, si-TUG1, si-TUG1 + miR-29c-NC, and si-TUG1 + miR-29c-3p inhibitor. Following incubation for $48 \mathrm{~h}$, RGS1 protein levels in A375 and SK-MEL-2 cells were detected by western blot assays. Representative results are shown from three repeats with a similar pattern. (D) Fold enrichment of miR-29c-3p after incubation with TUG1 probe in the cells. The data are presented as the mean \pm standard deviation; ${ }^{* *} \mathrm{P}<0.01$. The comparisons were made by one-way analysis of variance followed by Dunnett's test. TUG1, taurine upregulated 1; RGS1, regulator of G-protein signaling 1.

A

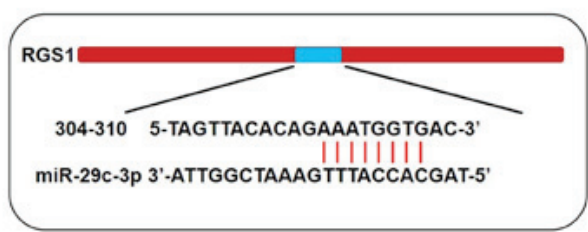

C

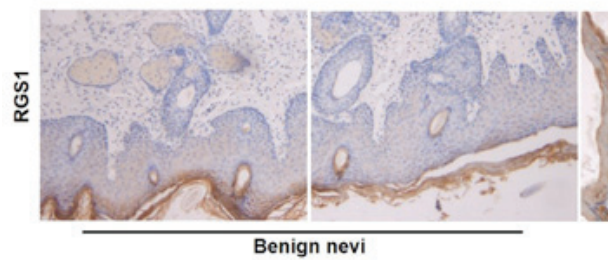

B

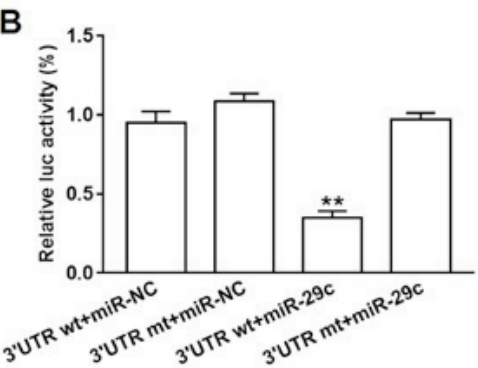

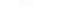
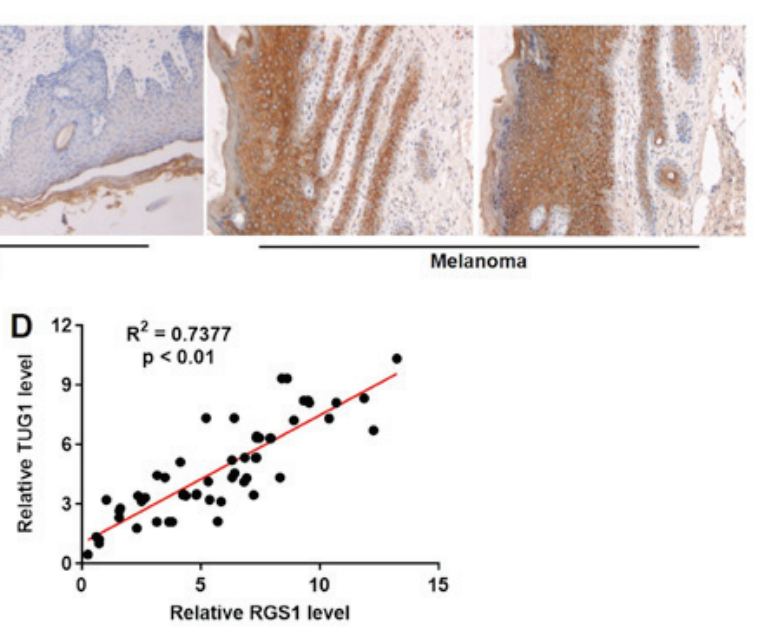

Figure 7. TUG1 regulates RGS1 expression via miR-29C-3p in melanoma cells. (A) Schematic representation of a miR-29c-3p seed matches the 3'-utranslated region (UTR) of RGS1 mRNAs as identified by TargetScan. (B) Luciferase activity in cells co-transfected with 3'-UTR wild-type (wt) + miR-NC, 3'-UTR mutant (mt) + miR-NC, 3'-UTR wt + miR-29c-3p, or 3'-UTR mt + miR-29c-3p. (C) The expression of RGS1 in human benign nevi and melanoma tissues was detected by immunohistochemical staining. The photographs were captured under a magnification of x200. (D) Pearson's correlation scatter plot of the fold change of TUG1 and RGS1 in human melanoma tissues. Representative results are shown from three repeats with a similar pattern. The data are presented as the mean \pm standard deviation. ${ }^{* *} \mathrm{P}<0.01$. The comparisons were made by one-way analysis of variance followed by Dunnett's test. TUG1, taurine upregulated 1 ; RGS1, regulator of G-protein signaling 1. 

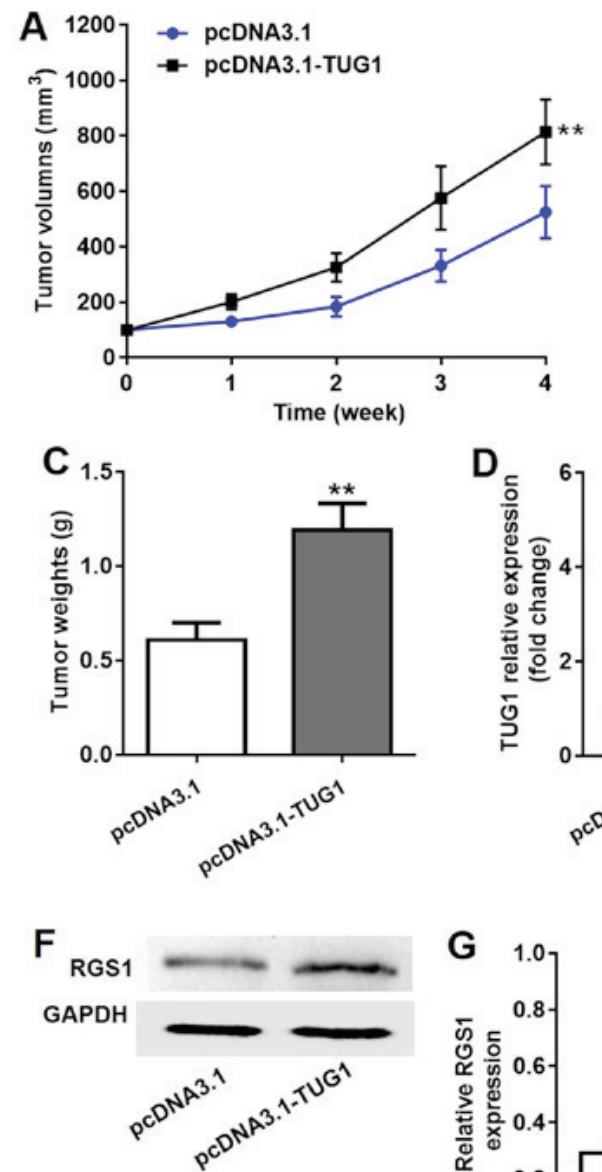

B

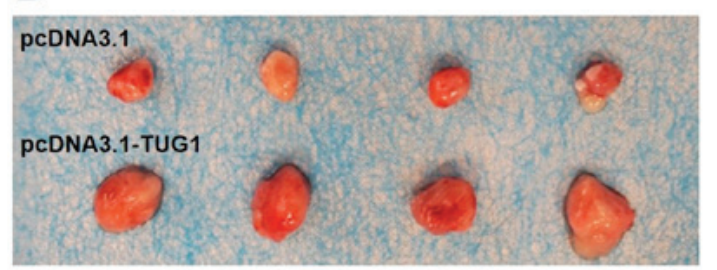

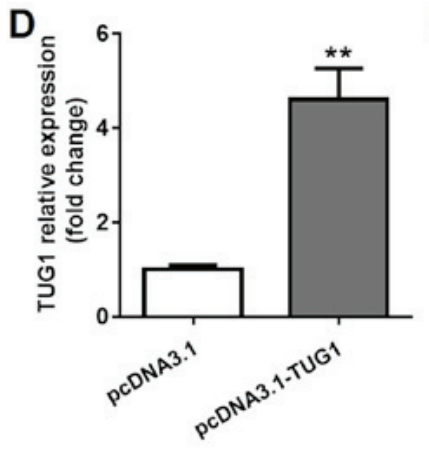
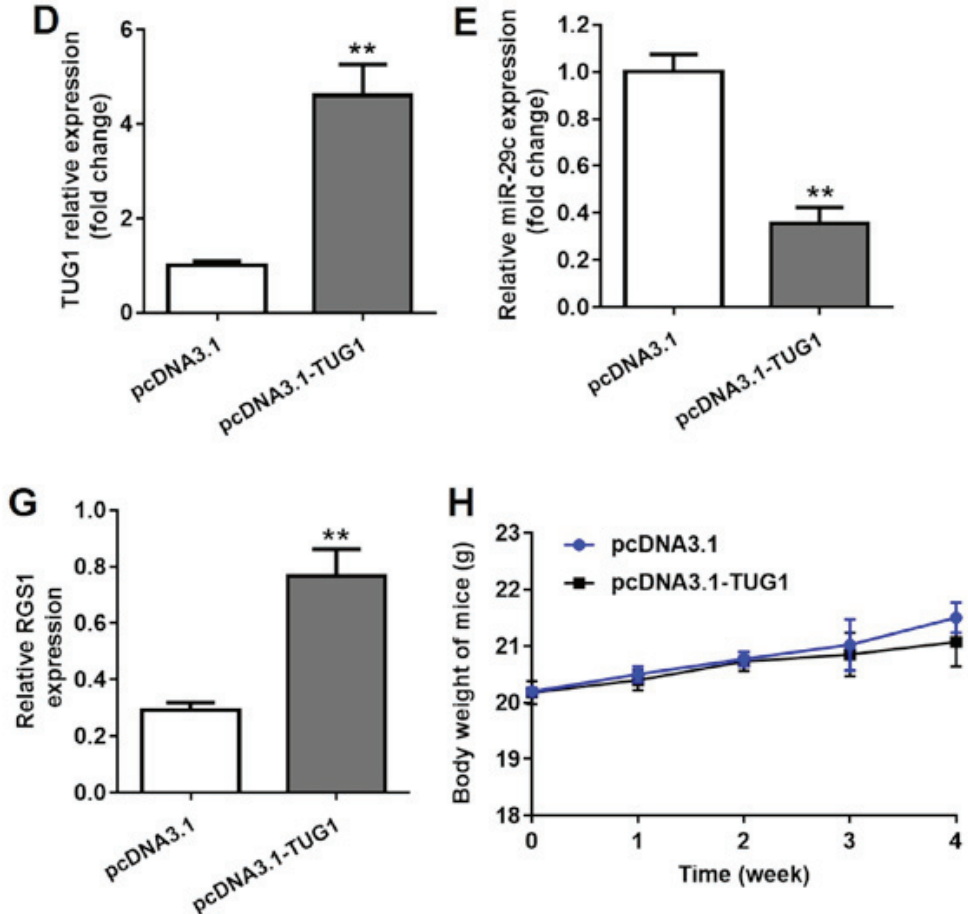

Figure 8. LncRNA TUG1 promotes A375 xenograft tumor growth in BALB/c nude mice. (A) Tumor volumes were measured once a week. (B) Tumor tissues were excised from the mice 4 weeks following transfection. (C) The mean tumor weight of the pcDNA3.1-TUG1 group was increased. (D and E) The relative expression levels of TUG1 (D) and miR-29c (E) in tumor tissues were detected by reverse transcription-quantitative polymerase chain reaction analysis (F and G) The protein RGS1 expression was detected by western blot analysis. (H) The body weight of mice was monitored weekly. The data are presented as the mean \pm standard deviation. ${ }^{* *} \mathrm{P}<0.01$. The comparison was made by Student's t-test. TUG1, taurine upregulated 1.

miR-29c-3p was precipitated by the TUG1 probe (Fig. 6D). These data revealed that TUG1 acted as a ceRNA to regulate the expression of miR-29c-3p and promote the expression of RGS1 in melanoma.

TUG1 regulates RGS1 expression via miR-29C-3p. The crucial role of miRNAs in regulating protein expression via posttranscriptional repression of mRNAs has been extensively investigated (31). In light of the above results, the TargetScan algorithm we used to determine whether RGS1 is the target of miR-29c-3p. RGS1 was found to be a prominent target of miR-29c-3p (Fig. 7A). To confirm that RGS1 is a direct target of miR-29c-3p, the full length 3'-untranslated region (UTR) fragments of RGS1 and corresponding mutant counterparts were cloned directly downstream of the firefly luciferase gene. miR-29c-3p transfection significantly suppressed the luciferase activity of the reporter with RGS1 wild-type 3'-UTR (Fig. 7B). IHC staining indicated that RGS1 was highly expressed in human melanoma tissues compared with benign nevi (Fig. 7C). Additionally, correlation analysis revealed that the TUG1 expression level was positively correlated to that of RGS1 (R2=0.7377, P<0.01) (Fig. 7D).

TUG1 promotes melanoma growth in BALB/c nude mice. To further evaluate the role of TUG1 in vivo, a subcutaneous tumor model was established. A375 cells were transfected with pcDNA3.1-TUG1 and injected into nude mice.pcDNA3.1-TUG1 cell-derived xenograft tumors grew faster compared with pcDNA3.1 cell-derived xenograft tumors (Fig. 8A). The mean tumor weight in the pcDNA3.1-TUG1 group was also significantly higher compared with that of the pcDNA3.1 cell-derived xenograft tumors (Fig. 8B and C). We further validated that TUG1 expression levels in tumor tissues of the pcDNA3.1-TUG1 group were considerably higher compared with those in the pcDNA3.1 group (Fig. 8D). Moreover, the expression of miR-29c was downregulated in the tumor tissues of the pcDNA3.1-TUG1 group (Fig. 8E), while the level of RGS1 was upregulated by pcDNA3.1-TUG1 group (Fig. 8F and G). Additionally, there was no statistically significant difference in the body weight of mice between the pcDNA3.1 and pcDNA3.1-TUG1 groups (Fig. 8H). 


\section{Discussion}

Numerous studies have recently demonstrated that lncRNAs play an important therapeutic role in a variety of human diseases, including cancer. Moreover, a number of lncRNAs have been found to be abnormally expressed in melanoma cell lines $(11,32)$. An increasing number of reports have revealed that the expression of IncRNAs is regulated in a tissue-specific manner. IncRNAs play specific roles in several pathological processes, including tumorigenesis, angiogenesis, invasion and metastasis (33). These characteristics make lncRNAs possible candidates as diagnostic or prognostic cancer biomarkers. Over the past few years, a number of lncRNAs have been identified. A total of 77 lncRNAs have been identified as significantly dysregulated in melanoma cell lines and in primary melanoma samples from patients (34).

TUG1 is a critical regulator of cell proliferation in several cancer types, such as glioblastoma (35), osteosarcoma (26), bladder cancer (36) and colorectal cancer (37). TUG1 participates in the proliferation, migration, invasion and apoptosis of cancer cells. However, the role of TUG1 in melanoma cell lines and primary melanomas remains unknown. The function of TUG1 and underlying mechanism of action in melanoma remain largely unknown. Thus, there is an urgent need to investigate the expression levels and elucidate the underlying regulatory mechanism of TUG1 in melanoma.

The data of the present study indicated that the expression of TUG1 was upregulated in melanoma cell lines and primary melanoma samples, and that this expression was correlated with poor overall survival. Moreover, we determined the role of TUG1 in melanoma progression. The findings were consistent with those of previous reports (38) and demonstrated that the knockdown of TUG1 suppressed cell proliferation and invasion and induced cell apoptosis in melanoma. The overexpression of TUG1 promoted the growth and invasion of melanoma cells, and inhibited the induction of apoptosis. The data indicated that TUG1 played an important role in melanoma, suggesting that it may act as an oncogene.

Although a large number of lncRNAs were reported to play critical roles in human malignancies, the underlying mechanisms by which IncRNAs modulate tumor progression remain unclear (39). Recently, lncRNAs have been reported to act as ceRNAs that downregulate the expression and activities of miRNAs. The downregulation of miRNAs can subsequently adjust the suppression of miRNA targets. It was hypothesized that TUG1 targets miRNAs in melanoma. In line with the previous reports, our results indicated that TUG1 acted as an endogenous sponge of miR-29c-3p, suppressing miR-29c-3p expression. Recent reports have revealed that miR-138-5p can suppress cervical (38) and pancreatic cancer (40) progression by targeting SIRT1. The TargetScan algorithm was used to identify whether RGS1 was the target gene of miR-29C-3p. It was observed that miR-29c-3p could reverse the inhibitory effect of TUG1 on melanoma cancer cell progression, which may be involved in the activation of RGS1. Taken together, these data indicated that TUG1 facilitated cell proliferation and invasion and suppressed apoptosis by regulating the miR-29c-3p/RGS1 axis in melanoma, suggesting that 1ncRNA TUG1 is a promising diagnostic marker for melanoma patients. The results further indicated that the regulation of RGS1 in melanoma by TUG1 required the activity of $\mathrm{miR}-29 \mathrm{C}-3 \mathrm{p}$.

In summary, the present study verified that TUG1 plays a key role in melanoma progression as an oncogene by promoting proliferation and invasion of melanoma cells. Mechanistically, the results of the present study revealed that TUG1 facilitated proliferation and invasion and suppressed the induction of apoptosis by regulating the miR-29c-3p/ RGS1 axis in melanoma. The results suggest that the lncRNA TUG1 may be a useful prognostic biomarker in melanoma patients, as well as a novel potential therapeutic target for melanoma, which may be further investigated in future experiments.

\section{Acknowledgements}

The authors gratefully acknowledge support from the $\mathrm{Ke} \mathrm{Wu}$ (Xinxiang Central Hospital) for the valuable suggestions.

\section{Funding}

No funding was received.

\section{Availability of data and materials}

The datasets used and/or analyzed during the present study are available from the corresponding author on reasonable request.

\section{Authors' contributions}

YW, GL and AL are responsible for sample collection, experiment design and execution. LR and KW analyzed and interpreted the experimental data. AL drafted the manuscript, reviewed and approved the final draft of this manuscript prior to submission. All the authors have read and approved the final manuscript.

\section{Ethics approval and consent to participate}

The research protocol was approved by the Ethics Committee of Xinxiang Central Hospital (approval no. 2017068801). The sample collection and surgical procedures were not harmful to the patients. Written informed consent was obtained from all patients. The research protocol conformed to the principles outlined in the Declaration of Helsinki. The animal experiments were fully approved by the Ethics Committee of Animal Experiments of the Xinxiang Central Hospital. In addition, the present study was strictly performed in accordance with the recommendations in the Guide for the Care and Use of Laboratory Animals of the National Institutes of Health.

\section{Patient consent for publication}

Not applicable.

\section{Competing interests}

The authors declare that they have no competing interests to disclose. 


\section{References}

1. Maddodi $\mathrm{N}$ and Setaluri V: Role of UV in cutaneous melanoma Photochem Photobiol 84: 528-536, 2008.

2. Siegel RL, Miller KD and Jemal A: Cancer statistics, 2015. CA Cancer J Clin 65: 5-29, 2015.

3. Siegel RL, Miller KD and Jemal A: Cancer Statistics, 2017. CA Cancer J Clin 67: 7-30, 2017.

4. Torre LA, Sauer AM, Chen MS Jr, Kagawa-Singer M, Jemal A and Siegel RL: Cancer statistics for Asian Americans, Native Hawaiians, and Pacific Islanders, 2016: Converging incidence in males and females. CA Cancer J Clin 66: 182-202, 2016.

5. Wu T and Du Y: IncRNAs: From basic research to medical application. Int J Biol Sci 13: 295-307, 2017.

6. Brown CJ, Hendrich BD, Rupert JL, Lafrenière RG, Xing Y, Lawrence J and Willard HF: The human XIST gene: Analysis of a $17 \mathrm{~kb}$ inactive $\mathrm{X}$-specific RNA that contains conserved repeats and is highly localized within the nucleus. Cell 71: 527-542, 1992.

7. Clark MB, Amaral PP, Schlesinger FJ, Dinger ME, Taft RJ, Rinn JL, Ponting CP, Stadler PF, Morris KV, Morillon A, et al: The reality of pervasive transcription. PLoS Biol 9: e1000625, discussion e1001102, 2011.

8. Zhang HM, Yang FQ, Chen SJ, Che J and Zheng JH: Upregulation of long non-coding RNA MALAT1 correlates with tumor progression and poor prognosis in clear cell renal cell carcinoma. Tumour Biol 36: 2947-2955, 2015.

9. Gao K, Ji Z, She K, Yang Q and Shao L: Long non-coding RNA ZFAS1 is an unfavourable prognostic factor and promotes glioma cell progression by activation of the Notch signaling pathway. Biomed Pharmacother 87: 555-560, 2017.

10. Li Z, Zhao L and Wang Q: Overexpression of long non-coding RNA HOTTIP increases chemoresistance of osteosarcoma cell by activating the Wnt/ $\beta$-catenin pathway. Am J Transl Res 8 : 2385-2393, 2016

11. Khaitan D, Dinger ME, Mazar J, Crawford J, Smith MA, Mattick JS and Perera RJ: The melanoma-upregulated long noncoding RNA SPRY4-IT1 modulates apoptosis and invasion. Cancer Res 71: 3852-3862, 2011.

12. Flockhart RJ, Webster DE, Qu K, Mascarenhas N, Kovalski J, Kretz M and Khavari PA: BRAFV600E remodels the melanocyte transcriptome and induces BANCR to regulate melanoma cell migration. Genome Res 22: 1006-1014, 2012.

13. Tang L, Zhang W, Su B and Yu B: Long noncoding RNA HOTAIR is associated with motility, invasion, and metastatic potential of metastatic melanoma. BioMed Res Int 2013: 251098, 2013.

14. Pasmant E, Sabbagh A, Vidaud M and Bièche I: ANRIL, a long, noncoding RNA, is an unexpected major hotspot in GWAS FASEB J 25: 444-448, 2011.

15. Rapicavoli NA and Blackshaw S: New meaning in the message: Noncoding RNAs and their role in retinal development. Dev Dyn 238: 2103-2114, 2009

16. Katsushima K, Natsume A, Ohka F, Shinjo K, Hatanaka A, Ichimura N, Sato S, Takahashi S, Kimura H, Totoki Y, et al: Targeting the Notch-regulated non-coding RNA TUG1 for glioma treatment. Nat Commun 7: 13616, 2016.

17. Jiang L, Wang W, Li G, Sun C, Ren Z, Sheng H, Gao H, Wang $\mathrm{C}$ and $\mathrm{Yu} \mathrm{H}$ : High TUG1 expression is associated with chemotherapy resistance and poor prognosis in esophageal squamous cell carcinoma. Cancer Chemother Pharmacol 78: 333-339, 2016.

18. Liu L, Chen X, Zhang Y, Hu Y, Shen X and Zhu W: Long non-coding RNA TUG1 promotes endometrial cancer development via inhibiting miR-299 and miR-34a-5p. Oncotarget 8 : 31386-31394, 2017.

19. Sun J, Ding C, Yang Z, Liu T, Zhang X, Zhao C and Wang J: The long non-coding RNA TUG1 indicates a poor prognosis for colorectal cancer and promotes metastasis by affecting epithelialmesenchymal transition. J Transl Med 14: 42, 2016.

20. Huang MD, Chen WM, Qi FZ, Sun M, Xu TP, Ma P and Shu YQ: Long non-coding RNA TUG1 is up-regulated in hepatocellular carcinoma and promotes cell growth and apoptosis by epigenetically silencing of KLF2. Mol Cancer 14: 165, 2015.
21. Ma B, Li M, Zhang L, Huang M, Lei JB, Fu GH, Liu CX, Lai QW, Chen QQ and Wang YL: Upregulation of long non-coding RNA TUG1 correlates with poor prognosis and disease status in osteosarcoma. Tumour Biol 37: 4445-4455, 2016.

22. Zhang EB, Yin DD, Sun M, Kong R, Liu XH, You LH, Han L, Xia R, Wang KM, Yang JS, et al: P53-regulated long non-coding RNA TUG1 affects cell proliferation in human non-small cell lung cancer, partly through epigenetically regulating HOXB7 expression. Cell Death Dis 5: e1243, 2014.

23. Iliev R, Kleinova R, Juracek J, Dolezel J, Ozanova Z, Fedorko M, Pacik D, Svoboda M, Stanik M and Slaby O: Overexpression of long non-coding RNA TUG1 predicts poor prognosis and promotes cancer cell proliferation and migration in high-grade muscle-invasive bladder cancer. Tumour Biol 37: 13385-13390, 2016.

24. Wang PQ, Wu YX, Zhong XD, Liu B and Qiao G: Prognostic significance of overexpressed long non-coding RNA TUG1 in patients with clear cell renal cell carcinoma. Eur Rev Med Pharmacol Sci 21: 82-86, 2017.

25. Zhang Q, Geng PL, Yin P, Wang XL, Jia JP and Yao J: Downregulation of long non-coding RNA TUG1 inhibits osteosarcoma cell proliferation and promotes apoptosis. Asian Pac J Cancer Prev 14: 2311-2315, 2013.

26. Yun-Bo F, Xiao-Po L, Xiao-Li L, Guo-Long C, PeiZ and Fa-Ming T: IncRNA TUG1 is upregulated and promotes cell proliferation in osteosarcoma. Open Med (Wars) 11: 163-167, 2016.

27. Li G, Liu K and Du X: Long Non-Coding RNA TUG1 Promotes proliferation and inhibits apoptosis of osteosarcoma cells by sponging miR-132-3p and upregulating SOX4 expression. Yonsei Med J 59: 226-235, 2018.

28. Liu H, Zhou G, Fu X, Cui H, Pu G, Xiao Y, Sun W, Dong X, Zhang L, Cao S, et al: Long noncoding RNA TUG1 is a diagnostic factor in lung adenocarcinoma and suppresses apoptosis via epigenetic silencing of BAX. Oncotarget 8: 101899-101910, 2017.

29. Li J, An G, Zhang M and Ma Q: Long non-coding RNA TUG1 acts as a miR-26a sponge in human glioma cells. Biochem Biophys Res Commun 477: 743-748, 2016.

30. Tay Y, Rinn J and Pandolfi PP: The multilayered complexity of ceRNA crosstalk and competition. Nature 505: 344-352, 2014.

31. Bartel DP: MicroRNAs: Target recognition and regulatory functions. Cell 136: 215-233, 2009.

32. Mazar J, Sinha S, Dinger ME, Mattick JS and Perera RJ: Protein-coding and non-coding gene expression analysis in differentiating human keratinocytes using a three-dimensional epidermal equivalent. Mol Genet Genomics 284: 1-9, 2010.

33. Fatica A and Bozzoni I: Long non-coding RNAs: New players in cell differentiation and development. Nat Rev Genet 15: 7-21, 2014.

34. Xie HW, Wu QQ, Zhu B, Chen FJ, Ji L, Li SQ, Wang CM, Tong YS, Tuo L, Wu M, et al: Long noncoding RNA SPRY4-IT1 is upregulated in esophageal squamous cell carcinoma and associated with poor prognosis. Tumour Biol 35: 7743-7754, 2014

35. Zhao Z, Wang B, Hao J, Man W, Chang Y, Ma S, Hu Y, Liu F and Yang J: Downregulation of the long non-coding RNA taurineupregulated gene 1 inhibits glioma cell proliferation and invasion and promotes apoptosis. Oncol Lett 15: 4026-4032, 2018.

36. Jiang $\mathrm{H}, \mathrm{Hu} \mathrm{X}$, Zhang $\mathrm{H}$ and $\mathrm{Li} \mathrm{W}$ : Down-regulation of lncRNA TUG1 enhances radiosensitivity in bladder cancer via suppressing HMGB1 expression. Radiat Oncol 12: 65, 2017.

37. Cao J, Han X, Qi X, Jin X and Li X: TUG1 promotes osteosarcoma tumorigenesis by upregulating EZH2 expression via miR-144-3p. Int J Oncol 51: 1115-1123, 2017.

38. Zhu J, Shi H, Liu H, Wang X and Li F: Long non-coding RNA TUG1 promotes cervical cancer progression by regulating the miR-138-5p-SIRT1 axis. Oncotarget 8: 65253-65264, 2017.

39. Cheetham SW, Gruhl F, Mattick JS and Dinger ME: Long noncoding RNAs and the genetics of cancer. Br J Cancer 108: 2419-2425, 2013.

40. Tian S, Guo X, Yu C, Sun C and Jiang J: miR-138-5p suppresses autophagy in pancreatic cancer by targeting SIRT1. Oncotarget 8 : 11071-11082, 2017. 